WILLIAM M. KANTOR

\title{
PROJECTIVE PLANES OF TYPE I-4: ADDENDUM
}

The paper 'Projective planes of type I-4' appeared in this journal (3 (1974), 335-346) without the galley proofs being corrected. The purpose of this addendum is to discuss a result which was to have been added in the proofs, and then to list corrections to the published version.

\section{AdDition}

I am grateful to T.Storer for calling my attention to the following result, which he attributes to L.J.Paige. Since Paige has informed me that he probably did not prove it, this theorem must be regarded as 'folklore'. Consequently, I will include a proof.

THEOREM. No neofield of order $n \equiv 15$ or $21(\bmod 24)$ can exist.

Proof. Suppose such a neofield $R$ exists. Since $3 \mid n,(1+1)+1=0$ by (4.12ii). Set $R^{\#}=R-\{0,1,-1\}$ and $n=24 k+6 i+3$ (with $i=2$ or 3).

We claim that $R^{\#}$ is partitioned into $4 k+i$ sets of size 6 , each of the form $\Sigma=\left\{a, b, a^{-1}, b^{-1}, a^{-1} b, b^{-1} a\right\}$ with $a+b=-1$. For, first of all $a+b=$ $-1, a^{-1}+a^{-1} b=-1$, and $b^{-1}+b^{-1} a=-1$ are equivalent, so each member of $\Sigma$ uniquely determines all others. (This requires (4.1).) Note that $a=0$ if $b=-1$ and $a=1$ if $b=1$, so $a, b \in R^{\#}$ implies $\Sigma \subseteq R^{\#}$. Thus, the sets $\Sigma$ partition $R^{\#}$. If $|\Sigma|<6$, then we may assume $a=b, a^{-1}, b^{-1}, a^{-1} b$, or $b^{-1} a$. These yield the respective contradictions $-1=a+a=-a ; a^{2}=1$; $1=a(-1-a)$, so $a^{3}=1$ by (2.7iv), whereas $3 \nmid n-1 ; a^{2}=b=-1-a$, so $a^{3}=1$ again; and $1=b^{-1}$. This proves the claim.

Let $R^{2}$ denote the set of squares of $R$. By (4.4), $\left|R^{2}\right|=1+(n-1) / 2=$ $12 k+3 i+2$. If $i=2$ then $4 \nmid n-1$, so $-1 \notin R^{2}$; if $i=3$ then $4 \mid n-1$, so $-1 \in R^{2}$. Thus, $\left|R^{2} \cap R^{\#}\right|=12 k+3 i-(i-2) \equiv 2 i+2(\bmod 4)$.

$R^{2} \cap R^{\#}$ is partitioned into the sets $R^{2} \cap \Sigma$. If $a, b \in R^{2}$, then $\Sigma \subset R^{2}$; if $a \in R^{2}$ and $b \notin R^{2}$, then $R^{2} \cap \Sigma=\left\{a, a^{-1}\right\}$; if $a, b \notin R^{2}$ then $R^{2} \cap \Sigma=$ $\left\{a^{-1} b, b^{-1} a\right\}$. Let $s$ be the number of sets $\Sigma \subset R^{2}$, so $4 k+i-s$ is the number of sets $\Sigma$ meeting $R^{2}$ twice. Then $\left|R^{\#} \cap R^{2}\right|=6 s+2(4 k+i-s) \equiv 2 i$ $(\bmod 4)$. This contradiction proves the theorem.

Several remarks are in order. Pankin [17], [18], rediscovered properties of the sets $\Sigma$, and used them for (5.10) and (5.12). The preceding proof did not use planarity (i.e., properties (iv) and (v) at the beginning of Section 2). However, results of J.R. Doner ('CIP Neofields and Combinatorial Designs', Ph.D. Thesis, U. of Michigan 1972) show that planarity is essential if similar arguments are to succeed: without planarity, the desired algebraic systems 
can exist for all orders $n \neq 10$ and $n \neq \equiv 0,6,12,15,18,21(\bmod 24)$, even satisfying (4.1) and having $R^{*}$ cyclic. One of Pankin's goals was, in fact, to find a way to use planarity in order to study the sets $\Sigma$.

Note that the case $n \equiv 21(\bmod 24)$ is eliminated by (4.13ii). It has been included in the theorem because a similar argument handles the case of planes of type I-3, of order $n \equiv 15$ or $21(\bmod 24)($ or even $n \equiv 0(\bmod 6))$, such that (4.1) holds. The only additional argument needed is as follows. Since $8 \nmid n-1$, $R^{*}$ has cyclic Sylow 2-subgroups, and hence has a normal 2-complement. Thus, $\left|R^{2}\right|$ is as before.

\section{CORRECTIONS}

p. 336,1 . 19. Read $X$ for $\overline{\mathscr{H}}$.

p. 337, after line 19, insert:

(v) If $R^{*}$ is Abelian, then $R^{*}$ has at most one subgroup of order 3 ([1]; [17]).

p. $338,1.1$. Read $F$ for $R$.

1. -9 . Read fields for yields.

p. 339, 1. 13. $\{(a, b) \in G \times G \mid b=a+1\}$

1. 15. The fourth sum is over all $g \in G_{1}$.

p. $340,1 .-5$. Read $m^{2}-1$ for $m-1$

p. 341,1 . -9 . (4.9).

1. -8 . $\operatorname{Read}\left(\frac{2}{n-1}\right)$ for $2 /(n-1)$.

p. $342,1 .-16$. Read $m+1$ for $n+1$.

p. $344,1.8 .(5.11)$.

1. $215.3 \mid n-1$.

p. $345,1: 21.1971$.

p. 346, Additional reference: M.D.Pankin, 'On Finite Planes of Type I-4', J. Alg. 27 (1973), 257-277.

Author's address:

William M.Kantor

University of Oregon,

Eugene,

Oregon 97403,

U.S.A.

(Received May 1, 1975) 